\title{
Adaptive mesh refinement computation of acoustic radiation from an engine intake
}

\author{
Xun Huang ${ }^{a}$, Xin Zhang ${ }^{a, *}$, Simon Keith Richards ${ }^{b}$ \\ a Aeronautics and Astronautics, School of Engineering Sciences, University of Southampton, \\ Southampton SO17 1BJ, UK \\ b GE Global Research Centre, Niskayuna, New York, NY 12309, USA
}

Received 21 February 2007; received in revised form 19 September 2007; accepted 20 September 2007

Available online 22 September 2007

\begin{abstract}
A block-structured adaptive mesh refinement (AMR) method was applied to the computational problem of acoustic radiation from an aeroengine intake. The aim is to improve the computational and storage efficiency in aeroengine noise prediction through reduction of computational cells. A parallel implementation of the adaptive mesh refinement algorithm was achieved using message passing interface. It combined a range of 2nd- and 4th-order spatial stencils, a 4th-order low-dissipation and low-dispersion Runge-Kutta scheme for time integration and several different interpolation methods. Both the parallel AMR algorithms and numerical issues were introduced briefly in this work. To solve the problem of acoustic radiation from an aeroengine intake, the code was extended to support body-fitted grid structures. The problem of acoustic radiation was solved with linearised Euler equations. The AMR results were compared with the previous results computed on a uniformly fine mesh to demonstrate the accuracy and the efficiency of the current AMR strategy. As the computational load of the whole adaptively refined mesh has to be balanced between nodes on-line, the parallel performance of the existing code deteriorates along with the increase of processors due to the expensive inter-nodes memory communication costs. The potential solution was suggested in the end.
\end{abstract}

(C) 2007 Elsevier Masson SAS. All rights reserved.

Keywords: Adaptive mesh refinement; Computational aeroacoustics; Parallel computation; Aeroengine

\section{Introduction}

Stringent noise regulation requirements for modern aircraft have promoted research into efficient and accurate numerical methods capable of predicting aircraft noise, e.g. engine intake noise radiation. The physical process of noise generation and radiation is governed by the Navier-Stokes equations. At present, a full numerical solution of noise generation, propagation and radiation process using the Navier-Stokes equations is prohibitively expensive. However, certain aspects of the noise propagation and radiation process can be modelled by linearised equations. For example, in the duct upstream of the rotor-stator region of an aeroengine, where nonlinear and thermal noise generation effects are minimal, the propagation of the rotor-stator noise can be studied using the linearised

\footnotetext{
* Corresponding author. Tel.: ++44 (0)2380 592321.

E-mail address: x.zhang1@soton.ac.uk (X. Zhang).
}

equations about the mean flow. A significant amount of research has been undertaken to develop theoretical and computational methods to predict engine tonal noise propagation and radiation. However, the development of a cheap and quick computational method is still a challenging job. Of the three main numerical approaches for engine duct noise propagation and radiation problems, boundary element (BE) methods [12] are confined to problems of acoustics through uniform mean flows; finite/infinite element (FE/IE) methods [1] are generally restricted to acoustic propagation through irrotational mean flows; and computational aeroacoustic (CAA) methods based upon the Euler or linearised Euler equations (LEE) are much general in terms of governing physics [19]. However, CAA methods are more expensive. Realistic engineering applications of CAA methods call for continuous research into efficient computational schemes/methods.

AMR is efficient and effective in treating problems with multiple spatial and temporal scales [3]. It represents compu- 
tational domain as hierarchal refinement levels and increases points per wavelength only in areas of interest. A given spatial error tolerance is achieved by recursively refining meshes. Subsequently a localised mesh of high grid resolution is distributed within an otherwise coarse mesh. The computational efficiency is improved by reducing the required number of computational cells. The operation of refinement could be operated either for each single cell [3], i.e. cell-structured AMR, or for each single block $[13,25]$ and called block-structured AMR.

For the block-structured AMR method, a computational domain consists of blocks with a predefined number of cells, e.g. $4 \times 4$ cells in each block. If any cell in one block requires refinement, the whole block is refined [13]. As a result the data structure is only maintained for blocks. It is well accepted that block-structured AMR requires less programming efforts and is computationally more effective than cell-structured AMR with respect to communication costs and memory requirements. In this work we extend our earlier effort [10] where a block-structured AMR code was constructed and tested against benchmark problems on rectangular meshes. In order to solve aeroacoustic problems of practical significance, e.g. acoustic radiation from a general aeroengine intake, the current code is extended to support body-fitted meshes and works on parallel machines using message passing interface (MPI) library.

The major objective of this work is to apply the AMR strategy to study acoustic radiation from a generic aeroengine intake. The case is governed by LEE and computed on a mesh that is adaptively refined according to the magnitude of perturbation pressure gradients that reflects the sound propagation procedure. The accuracy of the prediction is compared with the earlier efforts [17-19]. Costs and the parallel speedup performance are given at the end of the paper.

\section{The parallel AMR algorithm}

On parallel machines, the existing AMR applications $[4,13,21]$ generally employ a block-structured AMR algorithm. It involves a) representing the two-dimensional (2D)/threedimensional (3D) hierarchical computational domain in blocks, b) connecting the generated blocks in a quadtree/octree data structure, c) estimating local truncation errors at all grid points and identifying blocks with excessive errors, d) regridding the identified blocks by superimposing or removing blocks to accommodate changes in flow physics, and e) redistributing computational load between processors to maintain dynamic load balancing. This procedure is operated recursively until either a given refinement/coarsening level is reached or a predefined local truncation error level has been met. After regridding, the initial conditions of the newly generated blocks are inherited from their base blocks. This operation is referred to as the AMR prolongation operation. Conversely, after each computing step, the solutions on the finer blocks should be used to update the solutions of the corresponding base blocks to maintain the desired accuracy. This is known as the AMR restriction operation. To solve partial differences of cells located near a block boundary, an extra area surrounding each block is required. This oper-

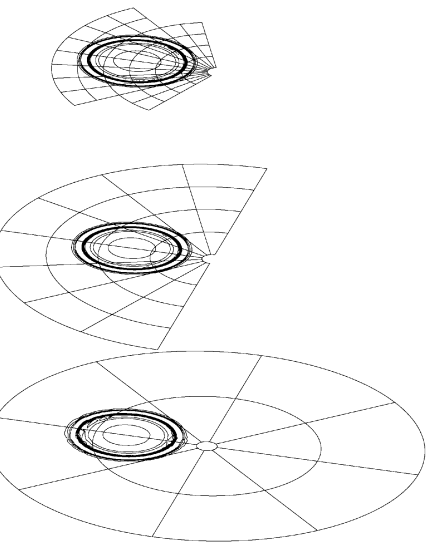

Fig. 1. Block-based AMR for an acoustic scattering problem. Solid lines denote blocks boundaries. Each block contains $21 \times 21$ cells.

ation is referred to as the ghost construction in the following description.

In Fig. 1 a schematic of the block-structured AMR method employed in this work is given. The example is a benchmark problem of acoustic scattering [22] solved with body-fitted multi-block AMR. The display style is chosen to illustrate the hierarchical structure of the adaptively refined mesh more clearly. In reality meshes on the fine levels are superimposed on the coarse meshes. In this example, a nested mesh consisting of three refinement levels is created at the start of the computation. The refinement ratio between the consecutive coarse and fine levels is two. The AMR regridding operation defines the relationships between the blocks as parents/children or sibling according to the type of their connection, stores the hierarchy information in the data structure of quadtree, and either refines or coarsens the hierarchical meshes based on the gradients of perturbation pressure. As the simulation progresses, the meshes are dynamically updated to reflect the evolving solution. The prolongation operation provides the initial solutions on the newly generated blocks, and the restriction operation updates the solutions on the coarse blocks.

The essential algorithm of the regridding operation is roughly the same in the existing programs [4,13], mainly consists of traversing in blocks to generate new blocks or to delete excessive blocks, and maintaining the corresponding data structure to reflect the volatile connection relationships between blocks. Nevertheless, the parallel strategies of the other AMR operations, i.e. prolongation, restriction and ghost construction are generally different. Fig. 2(a) illustrates the potential situations, where unidirectional communications are operated to transfer the whole solutions on the related blocks for prolongation and restriction, whereas bidirectional communications are used to transport a part of solutions on the corresponding blocks for ghost construction. To achieve high efficiency, separate communication subroutines were designed specifically for these AMR operations in the existing AMR programs [4,13], which consequently tend to be complex and inaccessible.

A simplified AMR library is developed as a starting point for our work in the area of CAA. To realise the parallel AMR operations as simply as possible, the essential decision is to combine the parallel communication of the AMR operations together 


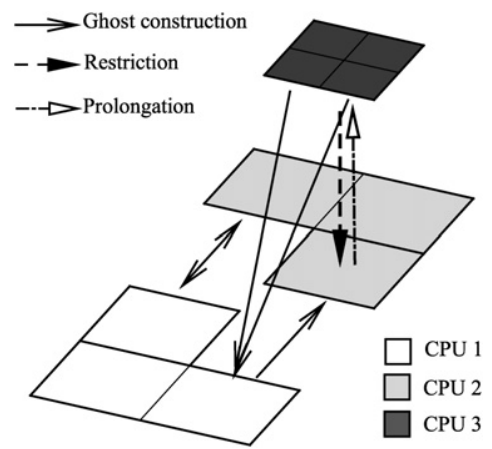

(a)

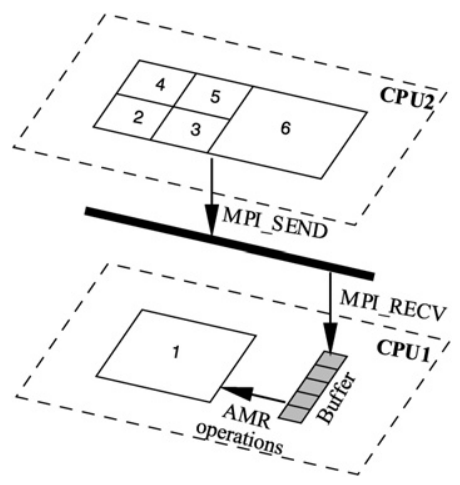

(b)

Fig. 2. Parallel AMR operations: ghost construction, restriction and prolongation.

to reduce the code complexity. A different example shows the basic idea in Fig. 2(b), where several blocks are assumed to be distributed on two processors (CPU1 and CPU2), and restriction and ghost construction are to be operated for block1 of Fig. 2(b). The simplified algorithm will set up a buffer on CPU1 by receiving all solutions of the related blocks $2-6$ of Fig. 2(b) on CPU2 prior to doing AMR operations for block1. Relying on the local buffer, the subsequent implementation of the AMR operations is an ordinary programming task. Compared to the existing AMR algorithms through a profiling tool, there is approximately $7 \%$ efficiency loss using the present simplified code for the interested CAA applications.

\section{Numerical issues}

A CAA algorithm includes ingredients such as high-order spatial stencils, temporal schemes, inflow, outflow and surface conditions. The examples presented in this paper use an explicit form of buffer zone [20] as the inflow, outflow and radiation conditions. To examine the global convergence rate on an adaptively refined mesh, the benchmark case of a Gaussian pulse propagation in a steady medium [6] is used. The problem is governed by the following equations:

$$
\begin{aligned}
& \frac{\partial u^{\prime}}{\partial t}+\frac{\partial p^{\prime}}{\partial x}=0, \\
& \frac{\partial v^{\prime}}{\partial t}+\frac{\partial p^{\prime}}{\partial y}=0, \\
& \frac{\partial p^{\prime}}{\partial t}+\frac{\partial u^{\prime}}{\partial x}+\frac{\partial v^{\prime}}{\partial y}=0,
\end{aligned}
$$

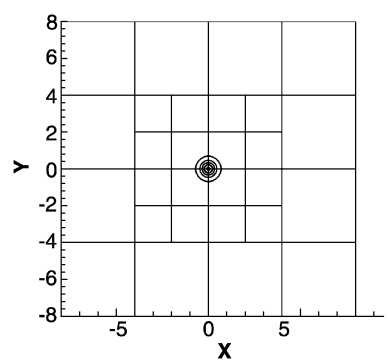

(a) $t=0.1$

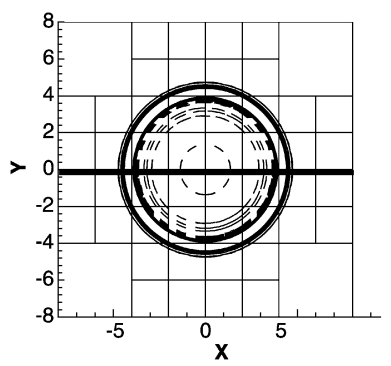

(b) $t=4$
Fig. 3. Perturbation pressure contours of 2D Gaussian pulse propagation. 16 equi-spaced contours between \pm 0.05 . Negative contours are dashed. Straight lines are blocks' borders. Solutions on the thick central line in (b) are used to compute the convergence rate.

with the initial condition given by

$p^{\prime}(x, y, 0)=\mathrm{e}^{-\left(\frac{x^{2}+y^{2}}{0.1}\right)}, \quad u^{\prime}(x, y, 0)=0$,

$v^{\prime}(x, y, 0)=0$,

where $x$ and $y$ are the Cartesian coordinates; $t$ is time; $u^{\prime}$ and $v^{\prime}$ are velocity perturbations in the $x$ and $y$ directions respectively; $p^{\prime}$ is pressure perturbation. The computation domain covers an area of $-8 \leqslant x \leqslant 8$ and $-8 \leqslant y \leqslant 8$.

The $2 \mathrm{D}$ problem is solved on an adaptive mesh that contains two refinement levels. Fig. 3 shows the wave propagation and the corresponding adaptively refined mesh. The Gaussian pulse is initiated at the centre of the mesh, where the finer mesh is superimposed to increase the resolution. The case is tested with a range of central spatial schemes, including 2nd- and 4thorder explicit schemes [16] and a 4th-order DRP scheme [24]. The 4th-order 4-6 low-dissipation and low-dispersion RungeKutta (LDDRK) [8] temporal scheme with constant time steps is used for time integration. A 10th-order filter [11] is employed throughout the domain to remove spurious waves. In the ghost construction operation, several interpolation methods (a 2ndand a 4th-order interpolation and a 6th-order polynomial interpolation) have been tested. The interval of mesh regridding is five computing steps, which is set according to the temporal step and the length of the ghost area, to guarantee that the wave propagation is always accurately resolved on the finest mesh.

The $L_{2}$-norm errors of the perturbation pressure are plotted in Fig. 4, where the cells number of each block ranges from $20 \times 20$ up to $50 \times 50$. The results show that the order of interpolation methods affects the convergence rate. The 2 nd-order interpolation keeps the 2nd-order convergence rate when combined with the 2nd-order explicit scheme. But it degrades the convergence rate to approximately 3 when the 4 th-order explicit scheme is employed. For general cases, Ray proposed to employ a 6th-order interpolation on an adaptively refined mesh to maintain the 4th-order convergence rate [15]. In his work a 36-points 2D interpolation was used. It is regarded as too cumbersome to implement for this work. As a result, the 6thorder polynomial interpolation is only operated separately in each coordinates direction. The convergence rate is increased to approximately 3.7 . 


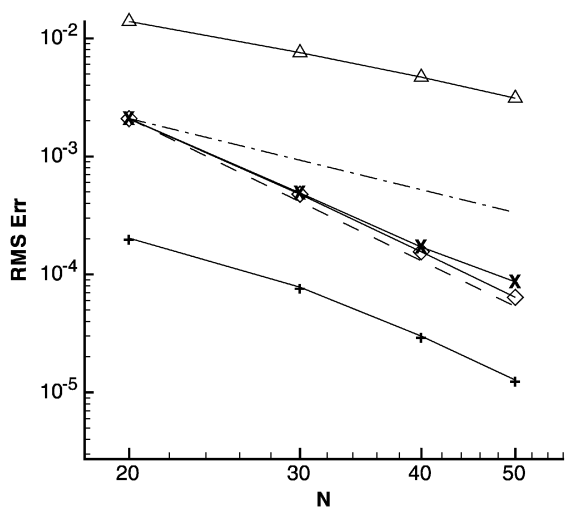

Fig. 4. $L_{2}$-norm error of the pressure, $\mathrm{N}$ is the number of cells in a block in one coordinate axis. $\triangle: 2$ nd-order explicit scheme and 2nd-order interpolation, $x: 4$ th-order explicit scheme and 2nd-order interpolation, $\diamond: 4$ th-order explicit scheme and 6th-order interpolation, +: 4th-order DRP scheme and 6th-order interpolation, --.: 2nd-order slope and - -: 4th-order slope.

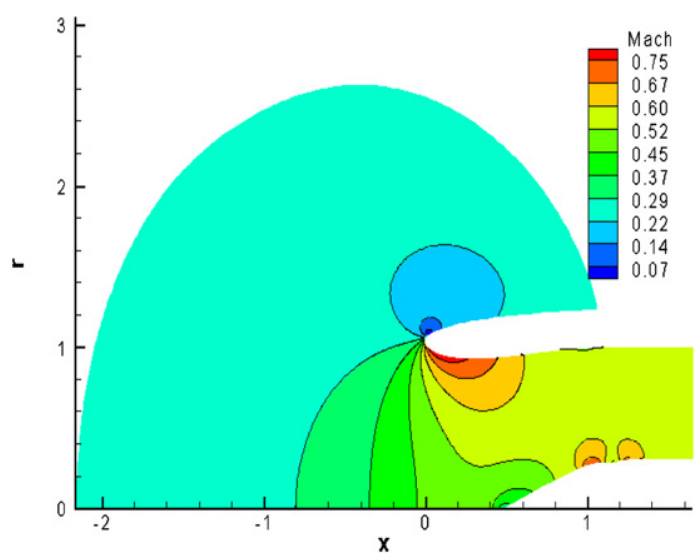

Fig. 5. Mach number contours of the mean flow around an aeroengine intake. Freestream Mach number is 0.25 , ambient pressure is $94250 \mathrm{~Pa}$, intake Mach number is set to 0.55 , and intake pressure is $79687 \mathrm{~Pa}$.

\section{Acoustic radiation from an engine intake}

In our previous work [10] the method of AMR was used in the computation of acoustic radiation along and away from an unflanged cylindrical duct. In this work the method is extended to a generic aeroengine intake with a realistic background mean flow, as shown in Fig. 5, where a high engine power setting is used. This section begins with an introduction of the governing equations and numerical methods. Calculations are based upon the radiation of realistic acoustic modes generated by the engine fan and fan/stator flow interactions. The computational results of the far-field directivity are to be compared with the results computed on a fixed mesh solving LEE and the predictions obtained from an established FEM solver [17]. The cost of AMR operations will be revealed by profiling the code on both the single- and multi-processors.

\subsection{Governing equations}

Assuming small perturbations about a steady mean flow, acoustic wave propagation can be described by LEE. Further, if the acoustic disturbances are restricted to the multiples of the blade passing frequency and propagate on an axisymmetric mean flow field without swirl, it is possible to write the disturbances in terms of a Fourier series, e.g. for the pressure disturbance $p^{\prime}$ at a single frequency $k$ the series is

$p^{\prime}(x, r, \theta, t)=\sum_{m=0}^{\infty} p_{m}^{\prime}(x, r) \mathrm{e}^{i(k t-m \theta)}$,

where $x$ is the axial coordinate, $r$ is the radial coordinate, $\theta$ is the circumferential angle, $t$ is time, and $m$ is the circumferential mode. Consequently, there are two important relations for the circumferential velocity disturbance $w^{\prime}$ and the pressure disturbance $p^{\prime}$ correspondingly. They are

$\frac{\partial w^{\prime}}{\partial \theta}=-\frac{m}{k} \frac{\partial w^{\prime}}{\partial t}, \quad \frac{\partial^{2} p^{\prime}}{\partial t \partial \theta}=m k p^{\prime}$.

The resulting set of equations are generally called 2.5D equations [26]. The complete governing equations in cylindrical coordinates for a single blade passing frequency $k$ are:

$$
\begin{aligned}
& \frac{\partial \rho^{\prime}}{\partial t}+\frac{\partial\left(\rho^{\prime} u_{0}+\rho_{0} u^{\prime}\right)}{\partial x}+\frac{\partial\left(\rho^{\prime} v_{0}+\rho_{0} v^{\prime}\right)}{\partial r} \\
& \quad-\frac{m \rho_{0}}{k r} w_{t}^{\prime}+\frac{\rho^{\prime} v_{0}+\rho_{0} v^{\prime}}{r}=0, \\
& \frac{\partial u^{\prime}}{\partial t}+u_{0} \frac{\partial u^{\prime}}{\partial x}+v_{0} \frac{\partial u^{\prime}}{\partial r}+\left(u^{\prime}+\frac{\rho^{\prime}}{\rho_{0}} u_{0}\right) \frac{\partial u_{0}}{\partial x} \\
& \quad+\left(v^{\prime}+\frac{\rho^{\prime}}{\rho_{0}} v_{0}\right) \frac{\partial u_{0}}{\partial r}+\frac{\partial p^{\prime}}{\rho_{0} \partial x}=0, \\
& \frac{\partial v^{\prime}}{\partial t}+u_{0} \frac{\partial v^{\prime}}{\partial x}+v_{0} \frac{\partial v^{\prime}}{\partial r}+\left(u^{\prime}+\frac{\rho^{\prime}}{\rho_{0}} u_{0}\right) \frac{\partial v_{0}}{\partial x} \\
& \quad+\left(v^{\prime}+\frac{\rho^{\prime}}{\rho_{0}} v_{0}\right) \frac{\partial v_{0}}{\partial r}+\frac{\partial p^{\prime}}{\rho_{0} \partial r}=0, \\
& \frac{\partial w_{t}^{\prime}}{\partial t}+u_{0} \frac{\partial w_{t}^{\prime}}{\partial x}+v_{0} \frac{\partial w_{t}^{\prime}}{\partial r}+\frac{m k}{\rho_{0} r} p^{\prime}+\frac{w_{t}^{\prime} v_{0}}{r}=0,
\end{aligned}
$$

where superscript (') and subscript (0) denote perturbation and mean properties respectively; $\rho^{\prime}$ is density perturbation; $u^{\prime}$ and $v^{\prime}$ are velocity perturbations in the $x$ and $r$ directions respectively; $w_{t}^{\prime}=\partial w^{\prime} / \partial t$. The fluid is modelled as a perfect gas with the homentropic assumption. $p^{\prime}=C_{0}^{2} \rho^{\prime}, C_{0}$ is sound speed. The boundary treatment for $w_{t}^{\prime}$ is the same as that for $w^{\prime}$.

The incident wave is defined as follows:

$$
\begin{aligned}
& \rho^{\prime}(x, r, \theta, t)= a\left[J_{m}\left(k_{r} r\right)+c_{1} Y_{m}\left(k_{r} r\right)\right] \cos \left(k t-k_{a} x-m \theta\right), \\
& u^{\prime}(x, r, \theta, t)= \frac{k_{a}}{k-k_{a} M_{j}} p^{\prime}, \\
& v^{\prime}(x, r, \theta, t)=-\frac{a}{k-k_{a} M_{j}} \\
& \times \frac{\mathrm{d}\left[J_{m}\left(k_{r} r\right)+c_{1} Y_{m}\left(k_{r} r\right)\right]}{\mathrm{d} r} \sin \left(k t-k_{a} x-m \theta\right), \\
& w_{t}^{\prime}(x, r, \theta, t)=-\frac{a m k\left[J_{m}\left(k_{r} r\right)+c_{1} Y_{m}\left(k_{r} r\right)\right]}{r\left(k-k_{a} M_{j}\right)} \\
& \times \sin \left(k t-k_{a} x-m \theta\right), \\
& w^{\prime}(x, r, \theta, t)= \frac{a m}{r\left(k-k_{a} M_{j}\right)} \\
& \times\left[J_{m}\left(k_{r} r\right)+c_{1} Y_{m}\left(k_{r} r\right)\right] \cos \left(k t-k_{a} x-m \theta\right),
\end{aligned}
$$


$p^{\prime}(x, r, \theta, t)=a\left[J_{m}\left(k_{r} r\right)+c_{1} Y_{m}\left(k_{r} r\right)\right] \cos \left(k t-k_{a} x-m \theta\right)$,

where $M_{j}$ is Mach number inside the duct; $a$ is fixed at $10^{-4}$ to ensure small relative changes in density (as required for LEE); $J_{m}$ and $Y_{m}$ are the $m$ th order Bessel functions of the first and second kind respectively; $k_{a}$ is the axial wave number and $k_{r}$ is the radial wave number. $k_{r}$ is the $n$th solution of the following equation determined by the hard-wall boundary conditions of the duct

$$
\begin{aligned}
& \frac{\mathrm{d}\left[J_{m}\left(y_{\text {outer }} k_{r}\right)\right]}{\mathrm{d} r} \frac{\mathrm{d}\left[Y_{m}\left(y_{\text {inner }} k_{r}\right)\right]}{\mathrm{d} r} \\
& \quad-\frac{\mathrm{d}\left[J_{m}\left(y_{\text {inner }} k_{r}\right)\right]}{\mathrm{d} r} \frac{\mathrm{d}\left[Y_{m}\left(y_{\text {outer }} k_{r}\right)\right]}{\mathrm{d} r}=0,
\end{aligned}
$$

where $y_{\text {outer }}$ and $y_{\text {inner }}$ are the height of the inlet duct inner wall and the inner hub radii in the inflow boundary. $k_{a}$ is calculated from

$k_{a}=\frac{k}{1-M_{j}^{2}}\left(-M_{j} \pm \sqrt{1-\frac{k_{r}^{2}\left(1-M_{j}^{2}\right)}{k^{2}}}\right)$,

the selection of plus or minus $( \pm)$ signs in the parenthesis is determined by the propagation direction of the spinning wave. Plus (+) is for the positive propagation direction in the axial coordinate, and vice versa. The constant $c_{1}$ satisfies the following relations

$c_{1}=\frac{\frac{\mathrm{d}}{\mathrm{d} r}\left[J_{m}\left(y_{\text {outer }} k_{r}\right)\right]}{\frac{\mathrm{d}}{\mathrm{d} r}\left[Y_{m}\left(y_{\text {outer }} k_{r}\right)\right]}$.

On the centreline boundary where $r=0$ a singularity exists. It is treated by using l'Hopital's rule to approximate $1 / r$ by $\partial / \partial r$ at the singularity. To solve these equations, 4 th-order spatial and temporal schemes $[8,16,24]$ are employed.

\subsection{Curvilinear coordinate system}

In an earlier work [9] it was shown that a Cartesian mesh with low-order immersed boundary method [14] performed much poorly than a body-fitted mesh to solve acoustic propagation problems with curved geometries. There are also other attempts of using AMR for body-fitted multi-block meshes $[2,21]$, where curved geometries were allowed to be transformed into and simulated using a uniform computational domain. This can be achieved by using the coordinate transformation given by Eqs. (12)-(14), which represent a transformation from the physical to the computational coordinates. For simplicity the time variance of both coordinate systems is not considered. It is worth noting that this part is not new and is just included to complete this work.

$\xi=\xi(x, r), \quad \eta=\eta(x, r)$.

The first order spatial derivatives of the governing equations are evaluated using the chain rule:

$\frac{\partial}{\partial x}=\frac{\partial \xi}{\partial x} \frac{\partial}{\partial \xi}+\frac{\partial \eta}{\partial x} \frac{\partial}{\partial \eta}$,
$\frac{\partial}{\partial r}=\frac{\partial \xi}{\partial r} \frac{\partial}{\partial \xi}+\frac{\partial \eta}{\partial r} \frac{\partial}{\partial \eta}$, with the transformation metrics defined as

$$
\begin{array}{lll}
\frac{\partial \xi}{\partial x}=J\left(\frac{\partial r}{\partial \eta}\right), & \frac{\partial \xi}{\partial r}=J\left(-\frac{\partial x}{\partial \eta}\right), \\
\frac{\partial \eta}{\partial x}=J\left(-\frac{\partial r}{\partial \xi}\right), & \frac{\partial \eta}{\partial r}=J\left(\frac{\partial x}{\partial \xi}\right) .
\end{array}
$$

$J$ is the transformation Jacobian relating the geometric properties of the physical space to the uniform computational space and is given by

$J=\left[\frac{\partial x}{\partial \xi} \frac{\partial r}{\partial \eta}-\frac{\partial x}{\partial \eta} \frac{\partial r}{\partial \xi}\right]^{-1}$.

The extra memory and computational costs required for the AMR code are the same as the modification costs for a nonAMR code.

\subsection{Far-field directivity}

For CAA methods, a finite computational domain is required, leading to the requirements of non-reflective acoustic boundary conditions and a radiation model, generally in the form of an integral representation. In this work, the far-field directivity is estimated via an integral surface solution of the Ffowcs Williams-Hawkings (FW-H) equation [5]. An integral solution of the FW-H equation is implemented numerically to allow the near and far-field noise levels to be determined efficiently. Fig. 6 illustrates a 3D FW-H integral surface around the engine duct. After several numerical experiments, it shows that the number of azimuthal grid points $Z_{\text {num }}$ satisfying $Z_{\text {num }} \geqslant 10 m$ ( $m$ is the circumferential mode) is enough to predict the far-field directivity accurately. The 2D solutions of Eqs. (7) are extended to the 3D surface by using Eq. (5), i.e. $p^{\prime}(\theta, t)=p^{\prime}(0, t+m \theta / k)$.

\subsection{Absorbing numerical noise}

To absorb high-frequency numerical errors unresolved by the employed spatial scheme, a 10th-order explicit filter [7] or

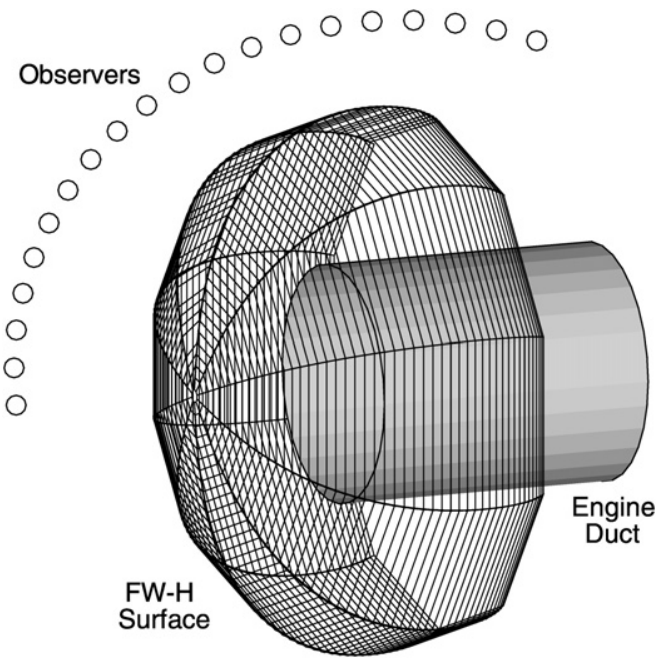

Fig. 6. 3D FW-H integral surface around the intake. 


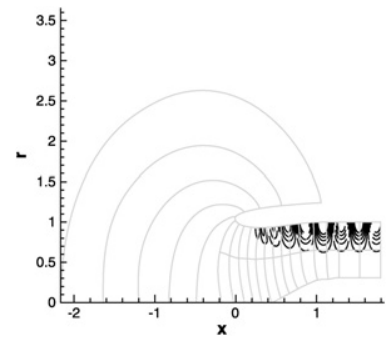

(a) Damping, $t=1.25$

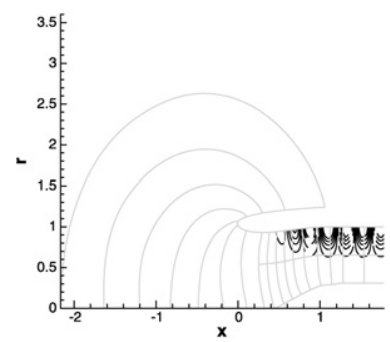

(c) Filter, $t=1.25$
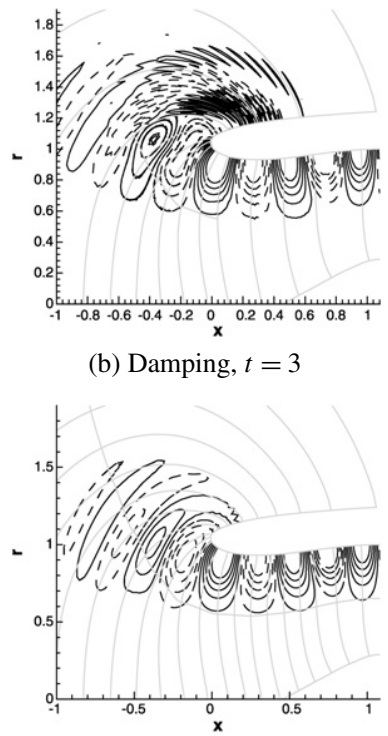

(d) Filter, $t=3$ (b) Damping, $t=3$

Fig. 7. Effect of spurious wave treatment methods. Perturbation pressure contours. Gray lines denote the boundaries of blocks. $m=12, n=1, k=1082 \mathrm{~Hz}$.

an artificial selective damping [23] was used in CAA applications. The 10th-order explicit filter takes the form:

$\overline{\chi_{i}}=\chi_{i}-\beta \sum_{k=0}^{5} a_{k}(\chi(i+k)+\chi(i-k))$,

where $\chi$ is the original variable, $\bar{\chi}$ the filtered solution, $\chi_{i}$ the filtered solution at the $i$ th gridpoint. The value of $a_{k}$ and $\beta$ can be found in literature [7]. Meanwhile, the discrete form of the scalar wave equation using the damping method at gridpoint $i$ can be written as:

$\left(\frac{\partial \bar{\chi}}{\partial t}\right)_{i}+\left(\frac{\partial \chi}{\partial x}\right)_{i}=-\frac{v_{a}}{(\Delta x)^{2}} \sum_{j=-3}^{3} d_{j} \chi(i+j)$,

where $\chi$ is the original variable, $\bar{\chi}$ the damped variable, $v_{a}$ an artificial kinematic viscosity, $j$ the damping stencil index, $d_{j}$ damping coefficients and $\Delta x$ the spatial discretisation size. The value of $v_{a}$ and $d_{j}$ can be found in literature [23].

Their effects are compared in Fig. 7, where the mesh is adaptively refined to capture the acoustic propagation. For the damping method the same coefficients used in a cavity flow simulation [23] are employed. Figs. 7(a)-7(b) show the method fails to absorb numerical noise generated around the lip of the intake. They suggest that the coefficients of artificial selective damping might have to be redesigned for the cases with curvilinear grids. Figs. 7 (c) -7 (d) indicate that the filter removes spurious waves more effectively. In the rest of the paper the 10th-order explicit filter [7] is employed to remove the spurious waves.

\subsection{Results and discussion}

With the aforementioned techniques, the acoustic propagation in and radiation from the aeroengine intake is solved with AMR. The coarsest block is composed of $17 \times 51$ cells to model the intake geometry accurately. In the computation, therefore,

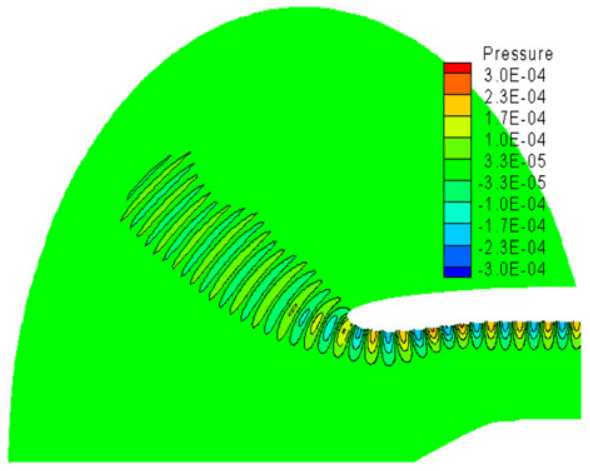

(a) LEE on a fixed mesh [17]

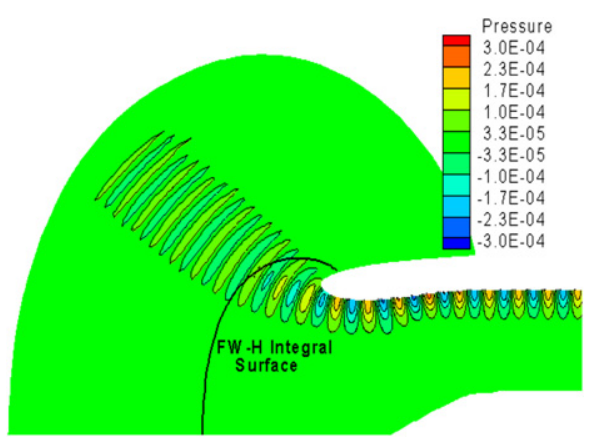

(b) LEE with AMR

Fig. 8. Perturbation pressure contours. $m=26, n=1, k=2268 \mathrm{~Hz}$. Setup without mean flow.

two to three refinement levels are enough to obtain satisfactory points per wavelength for the interested spinning modes. Once the waves reach the outflow boundary of the computation domain, the finest blocks span the whole computational domain and the AMR regridding operation could be stopped to improve efficiency. After that junction, the computation only proceeds on the top level blocks to save computational costs.

Two configurations are used: one with the steady medium and the other has the aforementioned realistic mean flow (Fig. 5). Figs. 8-9 compare the results of instantaneous perturbation pressure computed on an adaptively refined mesh with the same predictions computed on a fixed mesh [17]. There is little difference between the two results in the case of the steady medium, whereas for the realistic mean flow case the radiation pattern is slightly influenced by the AMR method. In order to show the difference much more clearly, the far-field directivity results computed with various strategies are compared in Fig. 10. The employed integration surfaces are already displayed in Figs. 8-9.

In the case with the steady medium, both the peak level and the peak radiation angle agree well with the results of Richards [17]. The peak radiation is predicted at $47.0 \mathrm{deg}$ (Fig. 10(a)). This compares well with the prediction $(47.27 \mathrm{deg})$ of Richards's work [17]. The dynamic range of the prediction is typically higher than $60 \mathrm{~dB}$ that is good enough for most of the engineering applications. The peak radiation magnitude is $0.55 \mathrm{~dB}$ lower than the result of Richard's work. It suggests that the filter might introduce an excessive level of dissipation surrounding the lip of the aero-engine intake. Meanwhile, the prediction does not follow the decaying envelope at low observation an- 


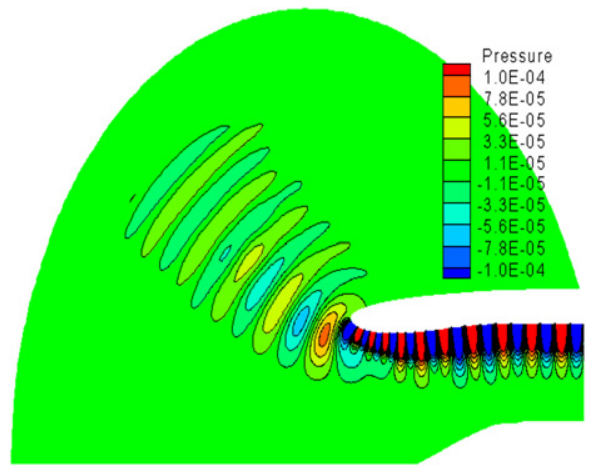

(a) LEE on a fixed mesh [17]

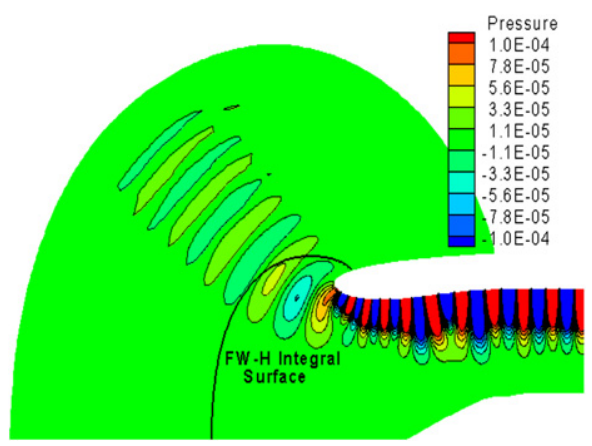

(b) LEE with AMR

Fig. 9. Perturbation pressure contours. $m=13, n=1, k=904 \mathrm{~Hz}$. Setup with mean flow.

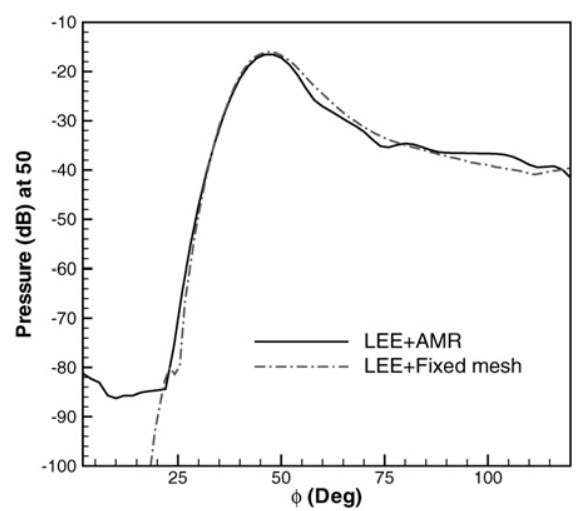

(a)

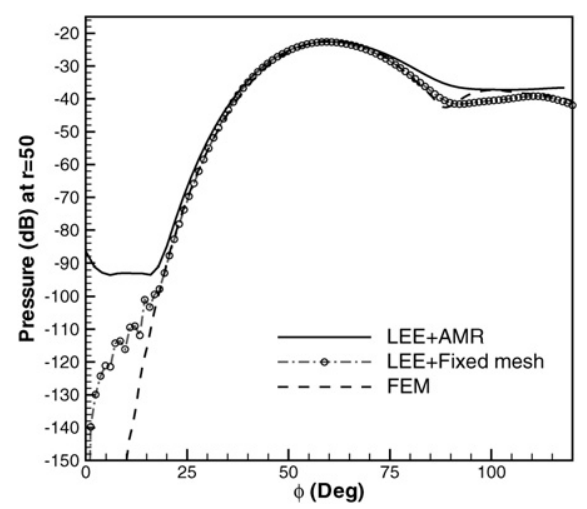

(b)

Fig. 10. Far-field directivities for the aeroengine intake, where (a) $m=26$, $n=1, k=2268 \mathrm{~Hz}$ without mean flow and (b) $m=13, n=1, k=904 \mathrm{~Hz}$ with mean flow. gles to the axisymmetrical axis $(\phi \leqslant 25 \mathrm{deg})$. The dynamic range of the prediction is somewhat smaller than the prediction of Richard. This is as expected as the order of the spatial scheme on an adaptively refined mesh is demonstrated earlier to be less than 4 , while the order of the spatial scheme employed in Richard's work was 6 . This particular feature might also be influenced by the spurious waves generated at the fine-coarse interfaces in the AMR operations. The accuracy also suffers slightly as the observation angle approaches $120 \mathrm{deg}$, the discrepancy in pressure level being at most $2.2 \mathrm{~dB}$.

For the realistic mean flow case, the results are presented in Fig. 10(b). The main peak angle and the peak level of the AMR result match the other two solutions well. The peak radiation angle is at $59.9 \mathrm{deg}$ (Fig. 10(b)) which compares favourablely with 59.4 deg predicted by Richard who solved LEE on a uniformly fine mesh and 60.8 deg by using an established FEM solver [17]. The differences of the peak radiation level between these results are less than $0.5 \mathrm{~dB}$, whilst the peak radiation angles differ from each other by less than $0.7 \mathrm{deg}$. Towards the low observation angle range $(\phi \leqslant 22 \mathrm{deg})$, the discrepancy in the pressure level increases again. The reason of this feature is the same as that explained in the previous case. The dynamic range of the prediction is also about $60 \mathrm{~dB}$. Nevertheless, the prediction deteriorates toward the high observation angles, especially around the shadow interference dip angles at $88.3 \mathrm{deg}$, where there is $7 \mathrm{~dB}$ difference between the AMR and the FEM results and $5 \mathrm{~dB}$ between the AMR and Richard's predictions on a fixed mesh. It could be caused by the spurious wave generated above the lip of the intake, as indicated by the wiggles shown in the perturbation pressure contours (Fig. 7(d)).

In the AMR computation of the steady medium case, the total number of cells increases from 13,872 to 41,616 as the wave propagates out of the intake. The computing time is $3463 \mathrm{sec}-$ onds on a desktop computer (Pentium IV $1.3 \mathrm{GHz}, 768 \mathrm{MB}$ ). In contrast, the computing time is 5400 seconds with the same AMR code working on a uniformly fine mesh without running the regridding operation. In an earlier computation with the SotonLEE code which also solves LEE [26], 7560 seconds is required to achieve the same results on a uniform mesh consisting of 81,600 cells. The corresponding computational time for the realistic mean flow case is 5904, 11,591 and 23,877 seconds, using the AMR code on the adaptively refined mesh, uniformly fine mesh and the SotonLEE code respectively. The results suggest that on a uniform mesh, the efficiency of the AMR code for the simpler steady medium case is lower than the efficiency of the SotonLEE code due to the extra cost of the AMR data structure management and the additional AMR operations, such as the ghost construction operation. In the relatively complex case with the realistic mean flow the similar AMR management cost is required for operating on the same mesh, while the computational cost for the LEE is increased extensively for solving the extra 12 convective terms so that leading to the comparable efficiency of the AMR code to the SotonLEE code on the uniformly fine mesh. Moreover, the results also suggest that the efficiency of the AMR code using adaptive meshes are up to $50 \%$ higher than the SotonLEE code. 


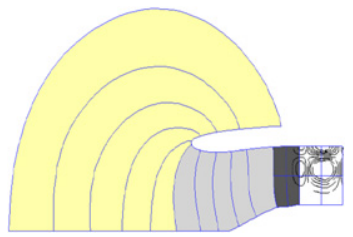

(a) 20 blocks, $t=0.566$

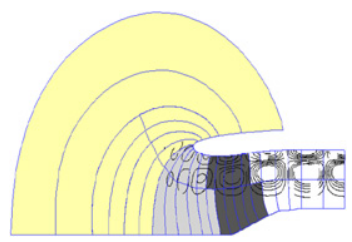

(c) 52 blocks, $t=2.83$

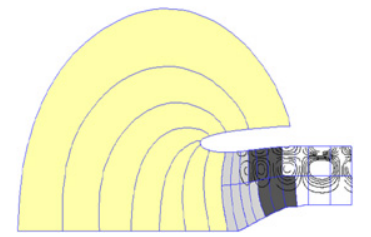

(b) 32 blocks, $t=1.698$

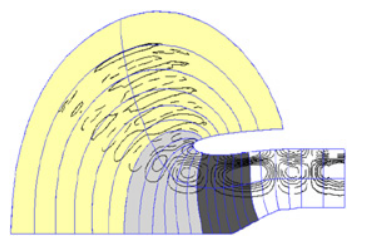

(d) 60 blocks, $t=11.32$
Fig. 11. Perturbation pressure contours, $m=12, n=2, k=20$. The computing load is distributed over 4 processors, which are denoted by different colours.

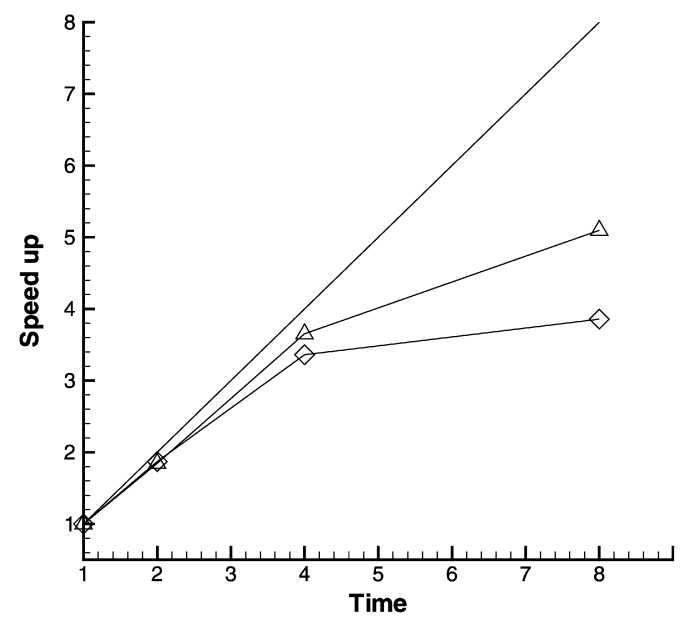

Fig. 12. The parallel speedup of the case of spinning mode sound radiation from an aero-engine intake with the realistic mean flow condition: (-) ideal speedup; $(\triangle)$ the result of the SotonLEE code; $(\diamond)$ the result of the AMR code.

Fig. 11 illustrates the process of regridding and dynamic load balancing within 4 processors which are differentiated by different colours. The computational load is redistributed evenly. In Fig. 12 the parallel speedup performance of the AMR code is compared with the parallel speedup performance of the SotonLEE code on a Beowulf PC cluster that consists of seven nodes connected by a Gigabit Ethernet. It is discovered that the communication cost of the AMR code is generally one to three times higher than the communication cost of the SotonLEE code. The cost is mainly contributed by the expensive communication cost of the AMR ghost construction operation. That operation consists of a lot of memory movements and network communications. Its performance is then limited by the present memory and network technology. Therefore, the speedup performance of the AMR method deteriorates slightly along with the increase of the communications. The parallel performance of the existing AMR code is still being optimised by exploiting the newly emerging multi-core technology with fast intra-node communication between parallel individual AMR processes.

\section{Summary}

Whereas the essential idea of AMR is not difficult to grasp, its parallel implementation is far from trivial. There exist many challenges in terms of algorithm development and computation. In this work the main AMR operations are explained and their parallel communications are bundled together to simplify the code developing efforts. The convergence rates of a range of 2nd- and 4th-order spatial schemes on an adaptively refined mesh are studied by solving the benchmark problem of Gaussian pulse propagation.

The developed AMR method is applied to the prediction of spinning modal radiation from a generic engine intake, with or without an axisymmetric mean flow. To model curved geometries, the AMR code is extended to support body-fitted grids. Filter and artificial selective damping are employed to absorb spurious waves generated in the AMR computation. Their effects are compared, and the filter method is shown to be the preferred method for the problem. The accuracy and the efficiency of the AMR method are demonstrated by the predicted far-field directivity, which compares well with those given by a LEE computation on a uniform mesh and FEM. In terms of computation efficiency, the adaptively refined mesh represents a saving of up to $50 \%$ compared with a uniform mesh. Relied on MPI, the computation loads are shown to be distributed evenly within the processors. The expensive communication cost in the AMR computation appears to be the bottleneck of the AMR parallel performance. In order to attain a higher efficiency on current parallel machines, it is suggested to separate the parallel communication of each AMR operation to obtain an optimal performance and exploit the newly emerging multi-core technology.

\section{References}

[1] R. Astley, J. Hamilton, N. Baker, E. Kitchen, Modelling tone propagation from turbofan inlets - the effect of extended lip liners, AIAA Paper 20022449, 2002.

[2] M.J. Berger, A. Jameson, Automatic adaptive grid refinement for the Euler equations, AIAA Paper 1985-1633, 1985.

[3] M.J. Berger, J. Oliger, Adaptive mesh refinement for hyperbolic partial differential equations, Journal of Computational Physics 53 (1983) 484512.

[4] P. Colella, D.T. Graves, T.J. Ligocki, D.F. Martin, D. Modiano, D.B. Serafini, B. Van Straalen, Chombo software package for amr applications, Lawrence Berkeley National Laboratory, 2003.

[5] J.E. Ffowcs-Williams, D.L. Hawkings, Sound generation by turbulence and surfaces in arbitrary motion, Philosophical Transactions of the Royal Society of London A 264 (1969) 321-342.

[6] J.C. Hardin, J.R. Ristorcelli, C.K.W. Tam (Eds.), Icase/larc Workshop on Benchmark Problems in Computational Aeroacoustics, NASA CP-3300, 1995.

[7] R. Hixon, Prefactored small-stencil compact schemes, Journal of Computational Physics 165 (2) (2000) 522-541.

[8] F.Q. Hu, M.Y. Hussaini, J. Manthey, Low-dissipation and low-dispersion Runge-Kutta schemes for computational acoustics, NASA CR-195022, 1994.

[9] X. Huang, X. Zhang, Parallel adaptive mesh refinement with boundary non-conforming grids, in: Euromech Colloquium 467, Marseille, France, July 2005.

[10] X. Huang, X. Zhang, Adaptive mesh refinement for computational aeroacoustics, AIAA Paper 2005-2873, 2005. 
[11] C.A. Kennedy, M.H. Carpenter, Comparison of several numerical methods for simulation of compressible shear layers, NASA Technical Paper 3484, 1997.

[12] S. Lidoine, H. Batard, H. Troyes, S. Delnevo, M. Roger, Acoustic radiation modelling of aeroengine intake comparison between analytical and numerical methods, AIAA Paper 2001-2140, 2001.

[13] P. MacNeice, K.M. Olson, C. Mobarry, R. de Fainchtein, C. Packer, Paramesh: A parallel adaptive mesh refinement community toolkit, Computer Physics Communications 126 (2000) 330-354.

[14] R. Mittal, G. Iaccarino, Immersed boundary methods, Annual Reviews of Fluid Mechanics 37 (2005) 239-361.

[15] J. Ray, C.A. Kennedy, S. Lefantzi, H.N. Najm, High-order spatial discretizations and extended stability methods for reacting flows on structured adaptively refined meshes, in: Proceedings of the Third Joint Meeting of the US Sections of the Combustion Institute, 2003.

[16] J. Ray, C.A. Kennedy, S. Lefantzi, H.N. Najm, Using high-order methods on adaptively refined block-structured meshes - discretizations, interpolations, and filters, SAND2005-7981, Sandia National Laboratories, 2006.

[17] S.K. Richards, Aeroacoustic computation of sound radiation from ducts, $\mathrm{PhD}$ thesis, School of Engineering Sciences, University of Southampton, 2005.

[18] S.K. Richards, X.X. Chen, X. Zhang, Computation of mode radiation from a generic aeroengine intake, in: European Congress on Computational Methods in Applied Sciences and Engineering ECCOMAS 2004, 2004.
[19] S.K. Richards, X.X. Chen, X. Zhang, Parallel computation of 3d acoustic radiation from an engine intake, AIAA Paper 2005-2947, 2005.

[20] S.K. Richards, X. Zhang, X.X. Chen, P.A. Nelson, Evaluation of nonreflecting boundary conditions for duct acoustic computation, Journal of Sound and Vibration 270 (2004) 539-557.

[21] J.S. Sachdev, C.P.T. Groth, J.J. Gottlieb, A parallel solution-adaptive scheme for predicting multi-phase core flows in solid propellant rocket motors, International Journal of Computational Fluid Dynamics 19 (2) (2005) $157-175$.

[22] C.K.W. Tam, J.C. Hardin (Eds.), Second Computational Aeroacoustics Workshop on Benchmark Problems, NASA CP-3352, 1997.

[23] C.K.W. Tam, K.A. Kurbatskii, Multi-size-mesh multi-time-step dispersion-relation-preserving scheme for multiple-scales aeroacoustics problems, International Journal of Computational Fluid Dynamics 17 (2) (2003) 119-132.

[24] C.K.W. Tam, J.C. Webb, Dispersion-relation-preserving finite difference schemes for computational acoustics, Journal of Computational Physics 107 (2) (1993) 262-281.

[25] A. Wissink, R. Hornung, Parallel amr application development with the samrai library, LLNL technical report UCRL-PRES-209469, 2005.

[26] X. Zhang, X.X. Chen, C.L. Morfey, P.A. Nelson, Computation of spinning modal radiation from an unflanged duct, AIAA Journal 42 (9) (2004) 1795-1801. 